\title{
El modelo para la capacitación de docentes en el sector de educación para personas sobredotadas, una investigación en América Latina
}

The Training Model of Teachers Within the Sector of Gifted Education, a Research in Latin America

O modelo de formação para docentes do setor de educação de pessoas sobredotadas, uma pesquisa na América Latina

\author{
Andrew Almazán-Anaya \\ Centro de Atención al Talento \\ Grupo Alianza Mexicana por la Sobredotación \\ Ciudad de México, México \\ almazananaya@cedat.com.mx \\ https://orcid.org/0000-0002-1498-6814 \\ Dafne Almazán-Anaya \\ Centro de Atención al Talento \\ Grupo Alianza Mexicana por la Sobredotación \\ Ciudad de México, México \\ daf.almazan@cedat.com.mx \\ https://orcid.org/0000-0001-6249-7049
}

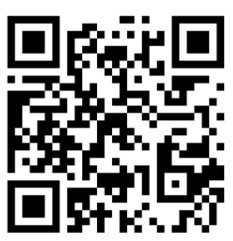

Recibido • Received • Recebido: 16 / $10 / 2019$

Corregido $\cdot$ Revised $\cdot$ Revisado: 19/ $06 / 2021$

Aceptado • Accepted • Aprovado: 28 / 07 / 2021

\begin{abstract}
Resumen:
Introducción. La educación del estudiantado sobredotado en América Latina todavía está en desarrollo, lo que incluye el proceso de capacitación de su profesorado, un aspecto clave en el éxito de las escuelas especializadas. Objetivo. Realizar un estudio que describiera y analizara el proceso de capacitación del personal docente para estudiantado sobredotado en tres de las escuelas diferenciadas (especializadas) más grandes de América Latina, con la finalidad de desarrollar una guía que reuna las experiencias en un plan general de capacitación. Metodología. La metodología fue cualitativa con entrevistas y observación de campo por cuatro años, en la que desarrollamos, a modo descriptivo, una guía por cada paso de la capaictación, basado en member checking, triangulación, verificación y cotejo con la bibliografía existente. Resultados, se describieron seis etapas de la formación del profesorado, lo que resultó en la obtención de un panorama general de cómo el profesorado de las escuelas especializadas en talento se capacitan en un país en desarrollo, donde los procesos formales y acreditados de formación docente aún no se han implementado completamente. Estos hallazgos mostraron fortalezas y debilidades en las etapas formativas de docentes en este ambiente especial que permiten mantener en funcionamiento centros educativos especiales de alumnado sobredotado
\end{abstract}


http://doi.org/10.15359/ree.25-3.15

http://www.una.ac.cr/educare

educare@una.ac.cr

en escuelas de América Latina, y creó una guía basada en la evidencia para la formación de futuros grupos de docentes en escuelas con estudiantes sobresalientes, especialmente cuando no hay un proceso de capacitación formal disponible en la localidad.

Palabras claves: Capacitación docente; educación de sobredotados; diferenciación; agrupamiento por habilidad.

\begin{abstract}
:
Introduction. The education of gifted students in Latin America is still developing; it includes the training process of its professors, a key aspect in the success of specialized schools. Objective. The objective was to conduct a four-year qualitative study with teachers, students, and principals to describe and analyze the teacher training process for gifted students in one of the largest differentiated (specialized) schools in Latin America. Method. The methodology was qualitative with interviews and field observation for four years; during this period, we developed a descriptive guide for each step of the training process based on member checking, triangulation, verification, and checking against the existing literature. Results. Six stages of teacher education were described, which resulted in obtaining a general panorama of how the teachers within gifted schools get trained in a developing country, where formal and accredited processes of teacher formation have not been fully implemented yet. These findings showed strengths and weaknesses in the formative stages of teachers in this special environment that allow keeping in operation special educational centers for gifted students in schools in Latin America. These findings also created an evidence-based guide for the training of future teachers in schools with gifted students, especially when there is no formal training process available locally.
\end{abstract}

Keywords: Teacher training; education of gifted; differentiated; ability grouping.

\title{
Resumo:
}

Introdução. A formação de estudantes talentosos na América Latina ainda está em desenvolvimento, o que inclui o processo de formação dos seus docentes, um aspecto fundamental para o sucesso das escolas especializadas. Objetivo. Realizar um estudo qualitativo de quatro anos com professores, estudantes e diretores, para descrever e analisar o processo de formação docente para estudantes talentosos em uma das maiores escolas diferenciadas (especializadas) da América Latina. Método. A metodologia foi qualitativa com entrevistas e observação de campo durante quatro anos, nos quais foi desenvolvido um guia descritivo para cada etapa do processo de formação, com base na verificação dos membros, triangulação, verificação e comparação com a literatura existente. Resultados. Foram descritas seis fases da formação de professores, que resultaram na obtenção de um panorama geral de como os professores das escolas dotadas recebem formação, num país em desenvolvimento, onde os processos formais e acreditados de formação de professores ainda não foram totalmente implementados. Estas descobertas mostraram pontos fortes e fracos nos estágios formativos da pessoa docente neste ambiente especial, que permitem manter em funcionamento centros educacionais especiais para estudantes superdotados nas escolas da América Latina, e criaram um guia baseado em evidências para a formação de futuros professores nas escolas com estudantes superdotados, especialmente em contextos em que não há processo de treinamento formal disponível localmente.

Palavras-chave: Formação de professores; educação de talentos; diferenças; agrupamento de capacidades. 
http://doi.org/10.15359/ree.25-3.15

http://www.una.ac.cr/educare educare@una.ac.cr

\section{Introducción y marco teórico}

La escolarización de estudiantado superdotado considerada parte de la educación especial, es una rama incompletamente desarrollada en todos los países, y se ha explorado poco sobre cómo capacitar a sus docentes (Barranco, 2012; Plunkett y Kronborg, 2011). La definición oficial de la National Association for Gifted Children (NAGC, 2010) considera al alumnado sobredotado como aquel que demuestra niveles sobresalientes de aptitud, con una habilidad excepcional para razonar y aprender, o con una competencia (desempeño o logro documentado en el top 10\%) en uno o más dominios (Ver Tabla 1 para definiciones oficiales utilizadas).

Tabla 1: Terminología oficial internacional utilizada en el estudio

\begin{tabular}{|c|c|}
\hline Término oficial & Definición \\
\hline Educación especial & $\begin{array}{l}\text { Rama de la educación enfocada en el alumnado con habilidades intelectual diferentes } \\
\text { al promedio (sobredotación intelectual o discapacidad), que lo hacen sujeto de } \\
\text { adecuaciones educativas especiales. Engloba todas las acciones a nivel educativo, } \\
\text { psicológico y pedagógico que se encaminan a compensar dichas necesidades. }\end{array}$ \\
\hline $\begin{array}{l}\text { Identificación (en } \\
\text { contexto educativo) }\end{array}$ & $\begin{array}{l}\text { Acción de reconocer que una persona guarda similitud. Compatibilidad con una guía } \\
\text { de características para realizar una comparación del objeto o persona observada en } \\
\text { relación con el modelo original. }\end{array}$ \\
\hline Inteligencia superior & $\begin{array}{l}\text { Sinónimo de sobredotación intelectual, término usado en psicometría para definir } \\
\text { al sector de la población con un percentil intelectual mayor a } 98 \text { (el cual únicamente } \\
\text { posee el 3\% de la población). }\end{array}$ \\
\hline $\begin{array}{l}\text { Sobredotación } \\
\text { intelectual }\end{array}$ & $\begin{array}{l}\text { Condición que un individuo presenta cuando posee un coeficiente intelectual } \\
\text { superior a los } 130 \text { puntos, según la Organización Mundial de Salud. }\end{array}$ \\
\hline $\begin{array}{l}\text { Enriquecimiento } \\
\text { académico }\end{array}$ & $\begin{array}{l}\text { Estrategia utilizada con niñez sobredotada que consiste en la complementación de la } \\
\text { enseñanza con el uso de material más avanzado al del grado cursado. }\end{array}$ \\
\hline Aceleramiento & $\begin{array}{l}\text { Estrategia consistente en adelantar uno o más grados académicos a niñez } \\
\text { sobredotada para prevenir su aburrimiento y estimular sus capacidades. }\end{array}$ \\
\hline $\begin{array}{l}\text { Diferenciación } \\
\text { educativa }\end{array}$ & $\begin{array}{l}\text { Término propuesto por Thomas Jefferson que engloba cualquier estrategia destinada } \\
\text { a separar en grupos homogéneos a estudiantes dependiendo de características } \\
\text { innatas o adquiridas para el uso posterior de educación especializada. Sinónimo de } \\
\text { educación proporcionada. En la sobredotación esto contempla la convivencia entre } \\
\text { iguales en alumnado sobredotado y el uso de clases adaptadas al nivel intelectual. }\end{array}$ \\
\hline
\end{tabular}

*Datos de la terminología basados en The International Test Commission Guidelines on computer-based and internet-delivered testing (Bartram, 2009), World Council for Gifted and Talented Children (Robinson, 2008) y la World Health Organization (2009).

Nota: Elaboración propia. 
http://doi.org/10.15359/ree.25-3.15

http://www.una.ac.cr/educare

educare@una.ac.cr

De acuerdo con los datos de los informes basados en los países de 2017 y 2018 del World Council for Gifted and Talented, en América Latina los escasos centros educativos especializados (diferenciados) utilizan metodologías validadas científicamente en proporción a los 12,9 millones de estudiantado sobredotado que debería vivir en la región. Se han utilizado modelos complementarios extraescolares con beneficios significativos para el estudiantado, aunque con una aplicación limitada en las clases y el seguimiento en comparación con los sistemas de tiempo completo (Almazán Anaya et al., 2014; Almazan-Anaya, 2021). Las personas investigadoras ya han descrito los efectos positivos de estos programas educativos diferenciados para estudiantes con inteligencia superior al promedio en el rango de beneficios psicológicos, académicos, intelectuales y sociales (Almazan-Anaya y Lozano-Rodríguez, 2015; Dai et al., 2011).

Sin embargo, el proceso que el personal docente requiere para atender a este estudiantado sobredotado apenas se aplica en varios países de América Latina. EI NAGC establece que un maestro o maestra dentro de este campo debe tener familiaridad con la teoría, la investigación, las estrategias curriculares y las prácticas educativas necesarias para desarrollar y mantener oportunidades de alta calidad en el aula para el aprendizaje avanzado del estudiantado (NAGC, 2013a). Las experiencias educativas en este campo son diferentes al promedio, ya que un estudiantado sobredotado tiende a requerir contenido más avanzado. En consecuencia, las estrategias de enseñanza y los contenidos académicos, incluida la intensidad de los programas, son diferentes de los experimentados en un aula de educación tradicional (Morales Silva, 2008).

Debido a la escasez de escuelas para alumnado sobredotado en todo el mundo, en comparación con el número de estudiantado talentoso, pocos proyectos de investigación han descrito el proceso de capacitación que atraviesa un maestro o maestra en el contexto de la educación de la niñez con inteligencia superior en un centro especializado (Plunkett y Kronborg, 2011; Whitton, 1997). El plan de estudios para estos grupos también es un desafío para el profesorado, por lo tanto, la capacitación que recibe es crítica. Hay institutos de primaria, secundaria y preparatoria de tiempo completo para este alumnado en todo el mundo, como la Academia Davidson en Nevada, la Gatton Academy en Kentucky, el Gifted Education Program en Singapur (Mandelman et al., 2010), el Centro de Atención al Talento (CEDAT) en México (Almazán Anaya, 2014), entre otros. El modelo específico aplicado en estas instituciones varía, aunque contemplan el enriquecimiento a través de la diferenciación, una estrategia con resultados favorables en función de la innovación, el liderazgo docente y la preparación de sus estudiantes y profesorado (Barranco, 2012; Vrignaud, 2006).

Estos programas especiales, reconocidos por su efectividad para el aprendizaje del estudiantado sobredotado (Kanevsky, 2011), emplean una diferenciación que consiste en evaluar al estudiantado en función de sus habilidades y potencial. Buscan que cada estudiante reciba, junto con sus pares, un desafío proporcionado o diferenciado, además de proporcionar el conocimiento avanzado requerido (Vrignaud, 2006). Los programas diferenciados no solo 
http://doi.org/10.15359/ree.25-3.15

son complejos para el estudiantado, sino también para el personal docente, ya que este último necesita integrar un rango de experiencias de aprendizaje en un nivel avanzado junto con habilidades de alto nivel e innovación educativa (Morales Silva, 2008). En consecuencia, no es infrecuente que el profesorado dude en implementar un programa educativo para estudiantado sobredotado para el cual no recibió preparación en sus estudios profesionales, debido a que rara vez reciben capacitación formal previa sobre educación para este tipo de estudiantes y la flexibilidad necesaria para adaptar el plan de estudios para estos grupos (Ghassib, 2010; Plunkett y Kronborg, 2011).

Según Vialle y Quigley (2003), los maestros y maestras que ingresan a escuelas diferenciadas deben cumplir requisitos específicos en relación con la capacidad intelectual y el conocimiento. En ciertos países, como los Estados Unidos, es necesario obtener créditos de un curso de certificación teórico-práctico o incluso un título de posgrado en el área para obtener una licencia en el campo de "Giftedness". En América Latina, siendo el tema de inteligencia superior menos avanzado, esta estructura administrativa es inexistente o escasamente desarrollada, por lo tanto, depende de cada institución para personas sobredotadas que sus docentes tengan la adecuada capacitación (Guerra Castillo et al., 2014; Mettrau, 2010). En México, este asunto es similar al resto de Latinoamérica, los centros educativos para alumnado sobredotado operan bajo principios de autocapacitación (el mismo centro entrena a sus docentes) y de experiencia de campo, pero sin una guía estandarizada del gobierno o un ente regulador. Todavía no está ampliamente documentado el proceso de capacitación requerido por docentes para estudiantado superdotado (Elices Simón et al., 2006; Mettrau, 2010; Tischler y Vialle, 2009; Whitton, 1997) ya que hay pocos manuales o planes definidos para este proceso educativo dentro de estos países.

El United States' Council for Exceptional Children y la NAGC en 2013 publicaron los Advanced Standards in Gifted Education Teacher Preparation remarcando las principales habilidades requeridas para profesionales que se involucran en el sector de estudiantado superdotado. Estos estándares abarcan modelos de evaluación, conocimiento del contenido curricular, facilitadores teóricos de programas y resultados, objetivos de liderazgo educativo y práctica ética profesional: modelos de colaboración. Estas mismas instituciones publicaron los Teacher Preparation Standards in Gifted and Talented Education que destacan las habilidades antes mencionadas y el entorno ideal para la formación de docentes (NAGC, 2013b). Debido a la naturaleza de estas normas, no son muy detalladas con respecto a la descripción de cómo debería ser este proceso de capacitación; se da por hecho la existencia de un sector educativo adaptado a este alumnado público y privado donde se evalúa al profesorado.

Por lo tanto, estas reglas se establecen como estándares ideales para docentes y sus habilidades esperadas dentro de este campo, donde funciona un sistema administrativo y educativo completamente funcional. En los países en desarrollo, no es raro que incluso las 
http://doi.org/10.15359/ree.25-3.15

http://www.una.ac.cr/educare

educare@una.ac.cr

autoridades educativas no puedan capacitar completamente a sus maestros y maestras de educación pública según lo previsto o no puedan evaluar rigurosamente sus habilidades antes de contratarles (Organización para la Cooperación y el Desarrollo Económicos [OCDE], 2010). Por lo tanto, un programa de capacitación estandarizado que siga únicamente las pautas de NAGC sería inviable en México, al carecerse, en ese sector, de guías o entes evaluadores con el rigor y experiencia requeridos para ese campo a nivel nacional. Además, se desconoce cómo estos mismos principios pueden aplicarse a los países en desarrollo (Feldhusen, 1997). Una investigación que en este campo permitiría uniformar, en el futuro, la serie de reglas profesionales hacia otros centros de estudiantado sobredotado con el fin de reducir los casos de inexperiencia por nuevos maestros y maestras y evitar un estigma social hacia la enseñanza del estudiantado sobredotado, como había sucedido en México (Almazán Anaya et al., 2014). La realización de más investigación en este campo permitiría crear un manual o guía basada en evidencia que, en ausencia de un estándar de capacitación docente a nivel local o federal, pudiera llenar ese vacío y servir para la formación de nuevo profesorado apto para incorporarlo en escuelas de alumnado sobredotado. En estos términos, encontramos que se requería un proyecto de investigación.

Como se reconoció anteriormente, el proceso de capacitación es un paso vital dentro de la educación de las personas sobredotados. Cabe señalar que este proceso no es medible, excepto por los puntajes obtenidos en pruebas de habilidad específicas; por lo tanto, no sería razonable trabajar únicamente con una investigación cuantitativa (Altrichter et al. 1993). Por lo tanto, en este artículo, se describe un estudio cualitativo, donde el objetivo principal consiste en el proceso de capacitación de docentes de estudiantado sobredotado en un ambiente de escuela diferenciada, para desarrollar una guía que reúna estas experiencias titulada Plan general de capacitación para profesores de estudiantes sobredotados.

\section{Metodología}

Realizamos un estudio cualitativo en el que se describió el proceso de capacitación de docentes entre enero de 2015 y diciembre de 2018. La investigación se realizó durante cuatro años en el Centro Mexicano de Alumnos Sobredotados (del sistema CEDAT, Centro de Atención al Talento), en su sedes ubicadas en la Ciudad de México, Guadalajara y Ciudad Satélite, actualmente la más grande institución en América Latina para estudiantado superdotado, y alberga a 50 docentes y más de 300 estudiantes con superdotación desde la primaria hasta la preparatoria. Veinte individuos fueron seleccionados. Esta muestra fue formada por personal directivo de la institución educativa, docentes senior con más de cinco años de experiencia (quienes a su vez estaban capacitando a nuevo profesorado en diferentes materias, como ciencias naturales y sociales, robótica e idiomas), docentes en formación y estudiantado sobredotado que había recibido clases de profesorado pertenecientes a este grupo. Realizamos observaciones de campo y entrevistas para recopilar información.

6 
http://doi.org/10.15359/ree.25-3.15

Utilizando estas técnicas, se recopiló información sobre las clases impartidas por los maestros y las maestras con su estudiantado superdotado durante un período de cuatro años, que buscaba describir la capacitación de los maestros y las maestras, sus actividades con sus estudiantes, cómo interactuaron con la clase, la respuesta del estudiantado, el tipo de tareas realizadas y el proceso de avance gradual de esta capacitación. Además, las personas investigadoras, a través de cuadrículas de observación directa, documentaron durante años estos datos, al describir y comparar las actividades mencionadas anteriormente. Para evitar interferir con el enfoque holístico de este proyecto, todas las entrevistas (realizadas una vez cada semestre) consistieron en preguntas abiertas que la persona investigadora hizo durante los días de observación en lugar de una entrevista formal de una o dos horas de duración. De esta manera, los sujetos participantes no fueron interrumpidos de sus actividades normales. Las preguntas se diseñaron para reunir los datos fundamentales para cumplir con los objetivos de la investigación, sin embargo, si una situación de capacitación específica no contemplada anteriormente fuera evidente, la entrevista podría ser dirigida a describir las situaciones emergentes. Asimismo, la investigación se realizó con la asesoría y guía de la Escuela de Graduados de Educación del ITESM Tecnológico Monterrey que supervisó el desarrollo de este proyecto de investigación para validación y análisis de datos.

\section{Participantes}

El estudiantado tenía entre 12 y 14 años al comienzo de este proyecto, equilibrado entre hombres y mujeres; a quienes se detectó previamente con sobredotación intelectual y acuden mínimo por 6 horas al día a clases presencialmente a centros de educación especial para recibir un programa diferenciado de atención. Este alumnado fue seleccionado por el método avalancha o bola de nieve de una población estudiantil cercana a los 100. Mientras que para el personal docente eran mujeres, su edad promedio era de 26 años, la mayoría de nacionalidades lationamericanas (hispanohablantes); de profesiones variadas como pedagogía, química, entre otros. El grupo también estaba integrado por personas directoras de la institución educativa analizada y maestros y maestras de más de cinco años de experiencia (quienes a su vez estaban capacitando al nuevo profesorado) en diferentes materias, como ciencias naturales, sociales, robótica e idiomas. Se seleccionaron dos maestros que estaban en un proceso de capacitación, así como tres alumnos y alumnas con sobredotación que habían recibido clases por parte de docentes que formaban este grupo.

\section{Validación}

Los estándares de rigor en el campo de la calidad señalados por Creswell (2013) indican que los datos obtenidos tienen que ser validados mediante la triangulación de la información (a través de análisis de contenido) y la verificación de miembros. Llevamos a cabo este proceso a través de 22 perspectivas diferentes (de los 20 sujetos participantes, el investigador y la 
http://doi.org/10.15359/ree.25-3.15

http://www.una.ac.cr/educare

educare@una.ac.cr

investigadora), asegurando un panorama más amplio sobre el objetivo del proyecto, que contempla analizar en qué converge, es distinto (o incluso se contradicen) cada una de las observaciones. Los hallazgos se organizaron en categorías basados en su complementariedad, exhaustividad y especificidad hacia: etapas de capacitación, características y vivencias de las etapas, y fortalezas/debilidades de la guía explicatoria del proceso de capacitación.

Se observaron aspectos éticos entre los que se encontró el consentimiento, la institución educativa, autorización de los padres y madres (en el caso del estudiantado) y una carta de conformidad por docentes y personal directivo participante. El código de ética que sirvió como guía fue el emitido por Harvard Graduate School of Arts and Sciences. Para mantener la privacidad, todos los nombres de participantes se mencionan como acrónimos.

\section{Resultados y discusión}

Después de cuatro años de investigación, describimos cualitativamente un proceso de capacitación para docentes de personas superdotadas en un país en desarrollo (México). En el contexto de esta investigación, la diferenciación se refiere a estudiantado superdotado en clases enriquecidas junto con personas que tienen inteligencia e intereses similares.

\section{El proceso de capacitación}

Describimos y observamos un modelo de capacitación que consta de seis fases diferentes que cada docente, después de ingresar a este campo educativo, experimenta en un período variable de tres a cinco años. En este análisis se consideró la integración de los criterios de especificidad y complementariedad, al resumir las características de cada fase del proceso de capacitación, así como las entrevistas y observaciones.

Estas etapas descritas conforman el cuerpo de una guía de capacitación, cada fase planteada fue nombrada, según lo sugerido por los sujetos participantes en las entrevistas, de la siguiente manera: detección de detección; fase de prueba de campo; profesorado asistido; enseñanza supervisada; docente superior y asesoría como tutor (ver Tabla 2). Investigadores e investigadoras anteriores que describieron los modelos de capacitación en los EUA encontraron una aproximación de entre cuatro y siete etapas que posteriormente fueron seguidas por nuevos maestros y maestras en escuelas especiales para personas sobredotadas (Feldhusen, 1997; Geake y Gross, 2008). Por lo tanto, nuestro modelo descrito tenía una estructura similar a las guías de capacitación anteriores definidas en otros países.

Sin embargo, en este estudio el investigador y la investigadora observaron cómo cada docente principal utilizó sus propias prácticas para capacitar a los nuevos maestros y maestras bajo su responsabilidad. Por lo tanto, inicialmente fue complicado describir una metodología en un proceso que variaba dependiendo de cada individuo. En esencia, tenían una estructura 
http://doi.org/10.15359/ree.25-3.15

http://www.una.ac.cr/educare educare@una.ac.cr

similar, pero varios sujetos profesores no informaron la justificación teórica subyacente. Por lo tanto, las autoridades escolares no pudieron rastrear inicialmente el progreso del proceso de capacitación de la manera eficiente, lo que sí hacían con el aprendizaje del estudiantado.

Tabla 2: Resumen del Plan integral de capacitación del personal docente de estudiantado sobredotado

\begin{tabular}{|c|c|c|c|}
\hline Etapa descrita & $\begin{array}{c}\text { Duración } \\
\text { aproximada }\end{array}$ & Objetivo principal & Características \\
\hline $\begin{array}{l}\text { 1. Detección de } \\
\text { candidatos/as (semana } \\
\# 0-1 \text { del ingreso) }\end{array}$ & $\begin{array}{l}\text { Menor a dos } \\
\text { semanas }\end{array}$ & $\begin{array}{l}\text { Preselección y evaluación } \\
\text { inicial de candidatos/as con } \\
\text { un perfil. }\end{array}$ & $\begin{array}{l}\text { Uso de pruebas psicométricas } \\
\text { para evaluar la inteligencia y } \\
\text { conocimientos. Entrevista. }\end{array}$ \\
\hline $\begin{array}{l}\text { 2. Prueba de campo } \\
\text { (semana \#3 a 6) }\end{array}$ & Un mes & $\begin{array}{l}\text { Depuración de la plantilla } \\
\text { de candidatos/as. }\end{array}$ & $\begin{array}{l}\text { "Filtro" de docentes, adquisición } \\
\text { de habilidades cognitivas y } \\
\text { desarrollo de un sentido de } \\
\text { humildad. }\end{array}$ \\
\hline $\begin{array}{l}\text { 3. Profesorado asistido } \\
\text { (semana \#7 a mes \#8) }\end{array}$ & $\begin{array}{l}\text { Un semestre (seis } \\
\text { meses) }\end{array}$ & $\begin{array}{l}\text { Adquisición de habilidades } \\
\text { básicas de enseñanza con } \\
\text { personas sobredotadas. }\end{array}$ & $\begin{array}{l}\text { Responsabilidad limitada, } \\
\text { docencia controlada por un tutor } \\
\text { y supervisor directo. }\end{array}$ \\
\hline $\begin{array}{l}\text { 4. Enseñanza supervisada } \\
\text { (Mes \#9 a \#18) }\end{array}$ & Un ciclo escolar & $\begin{array}{l}\text { Aprendizaje y transición } \\
\text { hacia las actividades de } \\
\text { profesorado. }\end{array}$ & $\begin{array}{l}\text { Desarrollo paulatino de todas } \\
\text { las actividades docentes, } \\
\text { tutoría indirecta, solo tiene tres } \\
\text { actividades restringidas. }\end{array}$ \\
\hline $\begin{array}{l}\text { 5. Docencia superior } \\
\text { o titular (A partir del } \\
\text { segundo ciclo escolar) }\end{array}$ & $\begin{array}{l}\text { Indefinido } \\
\text { (aproximado de } 5 \\
\text { a } 40 \text { años) }\end{array}$ & $\begin{array}{l}\text { Culminación de la } \\
\text { capacitación docente, } \\
\text { aplicación práctica de } \\
\text { conocimientos. }\end{array}$ & $\begin{array}{l}\text { Última fase en la mayoría de } \\
\text { los casos, asignación de grupo } \\
\text { dependiendo de la inteligencia } \\
\text { del grupo y del profesorado. }\end{array}$ \\
\hline $\begin{array}{l}\text { 6. Asesoría como tutoría } \\
\text { (A partir del año \#5) }\end{array}$ & $\begin{array}{l}\text { Indefinido (A partir } \\
\text { del quinto año de } \\
\text { la capacitación) }\end{array}$ & $\begin{array}{l}\text { Asesoría, con una } \\
\text { amplia transferencia } \\
\text { de conocimientos y } \\
\text { habilidades a nuevas } \\
\text { generaciones de docentes } \\
\text { en formación }\end{array}$ & $\begin{array}{l}\text { Fase voluntaria y especial (no } \\
\text { todos la alcanzan), permite la } \\
\text { renovación docente }\end{array}$ \\
\hline
\end{tabular}

Nota: Elaboración propia.

Se observó que los entes entrenadores notificaron a las autoridades cuando consideraron que los nuevos cuerpos docentes estaban listos para comenzar sus tareas de tiempo completo por su cuenta. Por lo tanto, fue complicado rastrear y comparar el modelo anterior utilizado entre el profesorado senior. Aún más, según un profesor senior "P.G", esta situación produjo una sobrecarga de deberes para el personal docente más experimentado: no estaba obteniendo suficiente tiempo para capacitar a un nuevo profesorado y cumplir con sus deberes con sus 
http://doi.org/10.15359/ree.25-3.15

http://www.una.ac.cr/educare

educare@una.ac.cr

estudiantes. Después de recibir cinco borradores autorreportados de modelos de capacitación diferentes por diferentes participantes, comenzamos a observar su aplicación general y notamos los pasos que definen cada etapa para permitir un seguimiento más fácil por parte del personal educativo y administrativo escolar. Durante los primeros meses se delineó un borrador donde las personas investigadoras comenzaron a trabajar gradualmente en la descripción de cada fase sobre un plan de capacitación unificado.

La primera fase descrita, que consta de los primeros días de entrenamiento, se denominó "Detección y detección de candidatos". El profesor R.H. (de Historia) describió esta etapa como "un proceso de evaluación psicométrica, que cumple un perfil de inteligencia, con el fin de que los aspirantes a maestros también estén dotados, conozcan el tema y las pruebas de personalidad". Se obtuvo un consenso y se validó observando los requisitos solicitados a cada persona candidata antes de su registro en el proceso de formación. La escuela les solicitó que pasaran las pruebas de inteligencia entre los primeros pasos antes del contrato. El requisito de talento de las personas candidatas se ha citado anteriormente en el campo donde los estudios indican que el profesorado de estudiantes de alta inteligencia probablemente expresarían un nivel cognitivo similar de acuerdo con sus estudiantes (Tischler y Vialle, 2009; Vialle y Quigley, 2002). Incluso aunque las pruebas de inteligencia pueden ser controvertidas, Clandinin y Connelly (1995) consideran que en los casos de selección de empleo donde las herramientas de evaluación son más confiables para fines de evaluación rápida, la selección de docentes basada en estas pruebas estaría justificada. En nuestra observación fue evidente que el objetivo era que cada docente tuviera suficientes conocimientos y habilidades profesionales, y que esto no resultara difícil de evaluar a primera vista sin el uso de pruebas estandarizadas.

En esta etapa, el profesorado superior citó el uso del proceso de selección como un paso necesario para regular quién ingresa a la escuela para comenzar a capacitarse. Este último se señaló como un factor crucial en este proceso porque, según el profesor principal de robótica):

Nuestros estándares de educación diferenciada permiten la convergencia de personas con las mismas habilidades, no solo de estudiantes sino de profesores. Quien esté a cargo de un niño con hiperactividad debe tener una noción básica del trastorno o incluso haber experimentado una situación similar para comprender mejor al estudiante. Con mucho talento es lo mismo, la alta demanda cognitiva de los estudiantes requiere que seamos igualmente capaces de responder o incluso más. De este modo, para nuestro sistema, un estudiante dotado requiere un profesor dotado. (J.R.)

Aún más, identificamos el uso de pruebas de conocimiento general, tales como exámenes profesionales de las áreas del personal docente (psicología, medicina, ingeniería, entre otros). Se agregó a estos hallazgos el hecho de que, durante la evaluación inicial, todas las personas candidatas son sometidas a una clase simulada de media hora acompañada por uno 
http://doi.org/10.15359/ree.25-3.15

de sus docentes senior. La clase consistió en algunos temas generales de ciencias donde el estudiantado también contribuyó (después de la clase) para compartir sus comentarios como coevaluadores del posible sujeto solicitante de empleo. En este último aspecto, la Directora Z.A. remarcó: es de suma importancia para los estudiantes superdotados que las instituciones reconozcan a los maestros prometedores que les gusta la clase escolar (sic) porque a veces estamos tratando de unirnos al personal que conoce la teoría de la diferenciación educativa. Este proceso tiene similitudes con lo que Tischler y Vialle (2009) describen como un perfil inicial preferido por el estudiantado sobredotado con respecto a sus docentes.

Se llamó a la segunda fase, por sugerencia del personal docente, fase de pruebas de campo, correspondiente al primer mes de capacitación. Observamos que los sujetos participantes discreparon con respecto a la forma en que se usa esta etapa debido a su impacto psicológico en el maestro o maestra, incluso si según la bibliografía es un paso recomendado. Todo el estudiantado consideró que era un momento difícil para el nuevo aprendiz, mientras que el profesorado senior y directivo coincidieron en la necesidad de utilizar esta etapa como un filtro. Según el profesor titular AM, esta etapa se utiliza principalmente para identificar la reacción del nuevo profesorado en condiciones de estrés y su respuesta a la responsabilidad. La observación directa confirmó que el objetivo de esta etapa es seleccionary capacitar a futuro personal docente en el campo, permitiéndole adoptar una velocidad, capacidad de resolución de problemas y estilo de vida compatible con la clase de estudiantes con inteligencia superior (que es más rápida que la tradicional). Debido a las demandas de esta etapa, se observó que la mitad de las personas candidatas suspendieron su entrenamiento y renunciaron, principalmente, debido al estrés psicológico y la ansiedad. Whitton (1997) describió que el proceso de enseñanza en este campo (instrucción para sujetos sobredotados) es complicado durante las primeras etapas, por lo tanto, esta fase puede ser parte de ese paso de adaptación.

A lo largo de varios meses, observamos que esta etapa involucraba una prueba práctica de resistencia de las autoridades escolares a sus nuevo profesorado. Según un profesor de idiomas:

Este mes impone un régimen de deberes doble a lo normal. El estrés y la ansiedad de los cambios hacen que cada uno exprese su verdadera personalidad y no la que un individuo finge en el trabajo ... Las defensas psicológicas y mentales se ralentizan, y los otros profesores o directores pueden analizar fácilmente las fortalezas y debilidades (sic) y así decidir quien posee el mayor potencial. (P.G)

El personal docente senior informó que al final de la fase de pruebas de campo, el futuro profesorado con un potencial más alto y cuyos resultados estaban de acuerdo con el ritmo del estudiantado sobredotado es contratado permanentemente. Observamos que el profesorado en esta etapa generalmente expresaba falta de conocimiento y experiencia en la enseñanza diferenciada, sin embargo, tenían una apertura al cambio. Este último factor ha sido identificado 
http://doi.org/10.15359/ree.25-3.15

http://www.una.ac.cr/educare

educare@una.ac.cr

por estudios anteriores como fundamental para la capacitación (Whitlock y DuCette, 1989). Según Vialle y Quigley (2003), el profesorado en formación debe volver a aprender sus técnicas de enseñanza, un proceso que requiere humildad para aceptar sugerencias y cambios (Lewis y Milton, 2005). El rasgo específico observado en esta etapa del personal docente es consistente con Mills (2003), quien informó una serie de requisitos básicos (que incluyen rasgos de personalidad) que el profesorado de estudiantes con sobredotación debe cumplir para tener éxito dentro de entornos de enseñanza diferenciados. Porque no era el propósito de este estudio describir un perfil docente dentro de la educación para estudiantado sobredotado, varios rasgos con respecto a la creatividad personal y las características sociales de este grupo de aprendices no se exploraron más.

Cabe señalar que la fase de pruebas de campo fue controvertida para los sujetos participantes, debido a su naturaleza como el momento más tenso en el proceso de capacitación. La directora ZA reconoció que el alumnado, especialmente cuando era joven, a veces experimentó trastornos del sueño durante todo este tiempo.

Los sujetos participantes subrayaron que exponer a un profesorado cansado en prácticas a varios grupos de estudiantes infantiles, incluso con supervisión, era contraproducente en su modelo educativo para estos grupos sobredotados. A.G. explicó este efecto:

los nuevos maestros tienen la misión de ocuparse de acciones básicas, como ayudarnos a participar más en clase, crear tareas y supervisar nuestros descansos. Cuando están cansados, no reconocen cuándo necesitamos asistencia o faltan para incluir algunas tareas diarias en nuestra sesión, entonces nuestras calificaciones pueden verse afectadas. (A.G.)

Además, los efectos académicos sobre estudiantes, producidos por sujetos aprendices cansados, fueron subrayados por estudiantes quienes no estuvieron de acuerdo con la necesidad de esta etapa. C.H. plantea que deja a los maestros más reflexivos, pero algunos empáticos y los que cuidan a los niños luego desaparecen. En consecuencia, detectamos que el grupo de estudiantes era el más opuesto a continuar esta etapa como un paso, ya que el profesorado con potencial o popular con el alumnado podría ser dado de baja del proceso de capacitación, no por falta de conocimiento o inteligencia, sino por su incapacidad para resistir esta fase de prueba. El personal directivo coincidió en que este filtro hizo un efecto en el número de candidaturas a docentes que pasó la primera etapa, pero falló en este paso, mencionó que la pérdida de la mitad de docentes fue considerable.

Cabe señalar que, en el estudio, esta fue una gran divergencia de opinión entre participantes y la bibliografía en este campo. Una deficiencia subrayada de este paso es la falta de apoyo psicológico para el nuevo profesorado y la flexibilidad de tiempo como lo notó Whitton (1997) en un proceso similar. En esta investigación, ambos directores respaldaron esta etapa con la condición de que el capacitador (un maestro experimentado) pudiera acortar el 
http://doi.org/10.15359/ree.25-3.15

tiempo de la etapa mencionada en función de sus propias evaluaciones. Docentes principales remitieron su sugerencia de mantener esa etapa de capacitación, pero evitar los efectos emocionales negativos y las altas tasas de deserción del alumnado. Finalmente, notamos que aquel profesorado que llega a la institución con experiencia laboral previa en otra escuela para alumnado sobredotado o que son estudiantes recientes (de programas para este alumnado) no tenía que pasar por la etapa de pruebas de campo o no experimentaba inconveniente alguno.

La tercera fase identificada en este estudio consistió en profesorado asistido, una etapa que dura aproximadamente un semestre en la que el nuevo profesorado siempre está con la supervisión de un maestro o una maestra con mayor experiencia. A través de la observación, descubrimos que este momento definió el comienzo del proceso formativo en el que el personal docente comenzó a recibir capacitación a través de un modelo de tutoría en técnicas de enseñanza para estudiantes con sobredotación en un entorno controlado.

Una observación del diario de una de las personas investigadoras con respecto a esta etapa fue:

El alumno sigue al maestro principal a cada clase ... En las dos horas de clase de robótica, el alumno tiene la responsabilidad de corregir y explicar los errores en los ensayos de cada alumno y coordinar la presentación de procedimientos de robótica en la sesión práctica. Los estudiantes y los nuevos maestros interactúan todo el tiempo, sin embargo, un profesor principal responde o interviene rápidamente cuando se hace evidente que el nuevo aprendiz está luchando para debatir alguna pregunta del grupo. (D.A.)

El equipo de trabajo analizó la fase de profesorado asistido, que fue definida por el Director AM: "una etapa que puede durar hasta un semestre ... Los nuevos maestros no son los responsables principales de las clases ya que siempre están bajo la supervisión de un senior profesor". Se describió que la función del profesorado en formación se enfoca en aprender de sus docentes con más experimentación y gradualmente trabaja con el estudiantado para establecer empatía y conocer su personalidad. Este es un proceso reforzado por los hallazgos de Mettrau (2010) que describió el trabajo del profesorado en un estudio sobre asesoramiento educativo y que analizó el "intercambio" de experiencias entre el profesorado. Sin embargo, en el presente estudio de investigación, identificamos que el personal docente auxiliar en este entorno escolar específico todavía tenía funciones limitadas y autoridad sobre el grupo, ya que no era responsable de responder al desarrollo general del estudiantado.

La cuarta fase se denominó enseñanza supervisada. En esta etapa encontramos un proceso gradual de desarrollo profesional, realizado durante el primer ciclo académico del profesor en la institución (después de terminar sus primeros seis meses desde su aceptación). Una gran parte de participantes acordó que en esta etapa a los maestros y maestras en capacitación ya se les ha asignado la mayoría de sus responsabilidades relacionadas con su puesto. Cabe señalar que en 
http://doi.org/10.15359/ree.25-3.15

http://www.una.ac.cr/educare

educare@una.ac.cr

esta etapa encontramos limitaciones específicas en las tareas del aula relacionadas más con las estrategias psicológicas. Por ejemplo, no se les permitió coordinar las evaluaciones y actividades de identidad intelectual (estrategias psicológicas y motivacionales a cargo de profesorado más experimentado o de otras áreas). A través de la observación de campo, se evidenció que el desempeño del profesorado en esta etapa se evalúa semanalmente y termina esta etapa con una ceremonia donde la comunidad lo reconoce como maestras y maestros talentosos que la comunidad escolar acepta. Este desarrollo bajo supervisión es un proceso gradual donde el nuevo profesorado adquiere las habilidades de enseñanza necesarias para trabajar en las aulas con estudiantado sobredotado.

Observamos equipos de docentes trabajando en una nueva estrategia para la integración de estos nuevos profesorados sin tener que otorgales todas las responsabilidades académicas que generalmente se dejan a los maestros y maestras senior. Cabe señalar que, en contraste con el sujeto mentor (un individuo que ya está a cargo de guiar a los nuevos grupos de docentes), el profesorado de tiempo completo es principalmente responsable de uno o varios grupos de estudiantes, mientras que las tareas del sujeto entrenador se centran más en los aprendices de docentes. En consecuencia, todas las personas participantes reportaron un fenómeno donde se implementaron nuevas tareas para los maestros y las maestras a tiempo completo que no están a cargo de capacitar a nadie: supervisar al profesorado en esta etapa por el motivo de que los requisitos de seguimiento son mínimos en comparación con las primeras etapas. Con esta medida, se observó cómo paulatinamente se liberó a los grupos mentores de docentes de una responsabilidad que ya no era obligatoria, permitiéndoles enfocarse en los primeros pasos de entrenamiento más críticos.

Una quinta fase descrita fue enseñanza superior, encontrada en este estudio como la culminación del proceso de capacitación para la mayoría del profesorado. Se observó que los maestros y maestras en esta etapa están asignados a un grupo que puede variar de 10 a 20 estudiantes con superdotación. Seis de los 20 participantes del grupo describieron en detalle que la edad promedio y el nivel académico del estudiantado que pertenece a cada aula dependen de las capacidades del personal docente, sus preferencias y habilidades. Estos factores remarcados por la investigación actual en este campo subrayan que tanto el estudiantado como el profesorado requieren ser compatibles (Bangel et al., 2010).

Se reveló una premisa presente en este proceso de capacitación: el compromiso de cada docente. El Director refirió:

siun nuevo profesorconsidera quees mejorcolocara los estudiantes dotados en un aula promedio o tiene objeciones contra la diferenciación por inteligencia, se convertirá en un obstáculo y, a su vez, la inversión destinada por la Institución educativa para la capacitación se perderá a medida que él (el profesor) renunciará a medida que aumenten sus obligaciones. (A.M.) 
http://doi.org/10.15359/ree.25-3.15

En este punto, el personal docente superior planteó la cuestión de la sobrecarga académica, que se hizo evidente al analizar esta etapa. Se observó que, por lo general, el profesorado senior enseñaba al estudiantado a tiempo completo y formaba a nuevos maestros y maestras. Según el profesor P.G.: Esta condición no permite suficiente tiempo para cumplir con nuestras obligaciones para con todos los estudiantes, tales como evaluaciones de supervisión, preparación de clases avanzadas y registro de informes académicos y sociales individuales.

Finalmente, se describió una sexta fase, que no todo el profesorado alcanza, la nominada: Mentoring as a Tutoro enseñanza como formador o formadora. Al final del período de observación, descubrimos que después de unos años (un mínimo de tres), los maestros y maestras con más experticia se involucran en la capacitación de otros grupos, por lo que este ciclo de formación se repitió con los nuevas personas candidatas. Un patrón de interés de esta etapa era alcanzado, en gran medida, por exalumnado graduado previamente de la institución diferenciada, quienes luego regresaron al centro educativo para convertirse en docentes (se crea, así, un sistema de autorrenovación del personal docente).

Como se describió anteriormente, la bibliografía en este campo mostró que a los sujetos entrenadores generalmente se les confieren diferentes tareas en comparación con el profesorado de tiempo completo, pues el primer grupo tiene que supervisar el trabajo de aprendices. Cabe señalar que entre el grupo de participantes no hay un acuerdo unánime en la definición de esta etapa porque la totalidad maestras y maestros no alcanzan estos niveles. El personal directivo entrevistado consideró innecesario describir una etapa que podría considerarse solo una extensión del paso de capacitación anterior. En cambio, el personal docente, en general, consideró que la causa principal de un aprendizaje eficiente era que existía un proceso organizado: alguien los entrena (a los maestros) y los últimos fueron entrenados también de antemano, por lo que deben ser fáciles de detectar como un nuevo tipo de miembros en nuestra escuela (J.R.). En contraste, el profesor principal J.R. consideró necesario reconocer este paso como una etapa específica que produce formadores de maestros en lugar de ser únicamente un profesorado de tiempo completo para estudiantado superdotado.

Después de describir este proceso a través de un trabajo continuo de observación y entrevista de cuatro años, concluimos la descripción de esta guía de capacitación para maestros y maestras en educación para alumnado sobredotado. Aún más, este modelo buscó capturar la descripción de cada participante del proceso de capacitación a través del autoexamen de su proceso de aprendizaje, además de las perspectivas externas de otras personas involucradas. Existen implicaciones directas para este proceso desde el proceso de entrenamiento cíclico. Un factor de interés fue la renovación del personal con exalumnado (maestros y maestras que regresaron y que se graduaron de escuelas especializadas). Se observó y analizó una tendencia de interés entre el estudiantado graduado: algunos alumnos alumnas regresan para convertirse en docentes. El profesor de historia y ciencias sociales agregó: 
http://doi.org/10.15359/ree.25-3.15

http://www.una.ac.cr/educare

educare@una.ac.cr

las instituciones más antiguas como los prusianos, ahora alemanes, tienen profesores con varias generaciones en sus instalaciones. Esto significa que sus estudiantes estaban preparados académicamente en un entorno diferenciado, continuaron su educación en el extranjero y posteriormente regresaron a impartir clases, lo que acelera el proceso de capacitación y promueve un compromiso de enseñanza. (B.D.G.)

Varios proyectos de investigación refuerzan esta afirmación al describir que este proceso de autorrenovación de la facultad se ha utilizado en varias de las escuelas más grandes para estudiantado superdotado del mundo, por ejemplo, la Escuela Federal Alemana de San Afra de Meisen (Barranco, 2012). En el futuro, este proceso podría contribuir, en gran medida, a mejorar el proceso de capacitación con la utilización de una estrategia efectiva donde un sujeto egresado ya sabe cómo funciona la escuela para que su adaptación como nuevo docente sea más rápida.

\section{Limitaciones de diseño e implicaciones del estudio}

Esta investigación cualitativa tiene un enfocado holístico. Sin embargo, puede carecer de aplicación hacia grandes generalizaciones. En consecuencia, los resultados de este estudio no pueden considerarse como un modelo internacional. Sin embargo, resultó en un paso hacia la descripción de una faceta de la educación para las personas con sobredotación por la creación de esta guía de capacitación en tres escuelas de este tipo.

La necesidad de crear esta guía de capacitación docente para escuelas de personas sobredotadas en países en vías de desarrollo era de larga data. Como se analizó a lo largo de los resultados, la falta de un proceso definido con frecuencia produjo pasos de capacitación mal dirigidos o en desoder que redujeron la efectividad del modelo educativo. Sugerimos que esta guía descrita permitiría a otras escuelas de alumnado sobredotado en América Latina y países en desarrollo analizar sus propios modelos de capacitación docente y mejorar la forma en que se organiza este proceso.

JR (docente de robótica) finalizó la última sesión de entrevista con una reflexión que resume la importancia de los hallazgos del proyecto: lo que se describió no es un proceso nuevo, (el modelo) ha estado en práctica desde hace varias décadas en otros países, pero pocos (centros diferenciados) los han analizado porque es parte de una cultura que no ha sido investigada por terceros y menos presentada a la comunidad científica.

Finalmente, describimos cómo los maestros y maestras en el entorno escolar de personas sobredotadas que estaban en una posición con capacidad de influir y producir cambios, a veces lo hacían porque las condiciones les obligaban, debido a la falta de una guía de capacitación formal. La desorganización escolar inicial en este proceso redujo su efectividad para estudiar y mejorarlo. Nuestra guía de capacitación podría ser un paso para solucionar ese problema para la autocrítica y proposición creativa. 
http://doi.org/10.15359/ree.25-3.15

\section{Conclusiones}

En este estudio se analizaron y describieron satisfactoriamente las fases de la formación de docentes en el contexto de la educación diferenciada para estudiantes con sobredotación, un proceso que no se investigó a fondo en América Latina antes, aunque de gran importancia para los servicios de educación especial en este sector. El modelo descrito se basa en las experiencias recabadas por entrevistas y observaciones de campo de varios participantes durante varios años en centros para alumnado sobredotado en México, y permitió observar el método autorregulado en capacitación de docentes en un entorno educativo especial. A través de esta investigación cualitativa, diseñamos una guía explicativa para la formación de profesorado con potencial para ser aplicado en las instituciones de estudiantado con inteligencia superior. Nosotros ampliamos y analizamos las fortalezas y debilidades de este nuevo Plan General de Maestros para la Capacitación en Escuelas de Sobredotados, a través de la rigurorsa realización de este proyecto cualitativo de cuatro años, donde exploramos seis etapas de entrenamiento docente. Este proceso de capacitación es, en parte, responsable del desempeño exitoso actual de los programas educativos diferenciados educativos en entornos nacionales donde no existe una supervisión y capacitación federal formal.

Sin embargo, todavía hay algunos temas para analizar, entre ellos: la influencia de estudiantes en la formación de profesorado de alumnado sobredotado. Observamos que entre los elementos que alentaron el desarrollo del profesorado, estaba la implementación de la diferenciación en sí misma, la ausencia previa de guías de enseñanza fijas para el profesorado y el trabajo extra producido por el hecho de acomodar la educación para estudiantado talentoso. ¿Podrían reproducirse estos factores en los centros educativos para estudiantes generales o solo pueden estar presentes en un entorno específico para estudiantes con sobredotación? Estas y otras preguntas sobre el mismo tema podrían ser una referencia para futuras investigaciones que centren su atención en estas preocupaciones educativas.

\section{Declaración de Material complementario}

Este artículo tiene disponible, como material complementario:

-La versión preprint del artículo en https://doi.org/10.5281/zenodo.4066969

\section{Referencias}

Almazan Anaya, A. (2014). The Psychoeducative Success Factors Within Differentiated Special Education for Gifted Students. US-China Education Review B, 4(11), 781-795. 
http://doi.org/10.15359/ree.25-3.15

http://www.una.ac.cr/educare

educare@una.ac.cr

Almazan Anaya, A. A. (2021). Efectos de la educación diferenciada en la inteligencia y el desempeño académico en estudiantado sobredotado en México. Revista Educación, 45(1), 1-18. https://doi.org/10.15517/revedu.v45i1.40401

Almazán Anaya, A., Dallal Fratz, I. y Accevo Zepeda, Z. (2014). Delegate Discourse. México. World Gifted. Newsletter of the World Council for Gifted and Talented Children, 32(2), 8-8. https:// world-gifted.org/publications/world-gifted-newsletter-archives/

Almazan Anaya, A. A., Illoldi Rangel, P. y Valdés Ramírez, M. del P. (2014). Educational Leadership within teaching of gifted children. Revista de Investigación Educativa de la Escuela de Graduados en Educación, 4(8), 25-34. https://www.researchgate.net/ publication/274736580 Educational Leadership in the teaching of gifted children

Almazan-Anaya, A. y Lozano-Rodríguez, A. (2015). El enfoque basado en competencias aplicado a estudiantes con sobredotación intelectual, su potencial en América Latina. Revista Electrónica Educare, 19(3), 1-23. http://dx.doi.org/10.15359/ree.19-3.14

Altrichter, H., Posch, P. y Somekh, B. (1993). Teachers investigate their work. An introduction to the methods of action research. Routledge.

Bangel, N. J., Moon, S. M. y Capobianco, B. M. (2010). Preservice teachers' perceptions and experiences in a gifted education training model. Gifted Child Quarterly, 54(3), 209-221. https://doi.org/10.1177/0016986210369257

Barranco, A. (2012). Investigación sobrela educación para niños con altas capacidades, flexibilización o enriquecimiento [Disertación doctoral]. Universidad Internacional de La Rioja.

Bartram, D. (2009). The International Test Commission guidelines on computer-based and internet-delivered Testing. Industrial and Organizational Psychology, 2(1), 11-13. https:// doi.org/10.1111/j.1754-9434.2008.01098.x

Clandinin, J. y Connelly, M. (1995). Teachers' professional knowledge landscapes. New York: Teachers College Press.

Creswell, J. W. (2013). Qualitative inquiry and research design: Choosing among five approaches. Sage.

Dai, D. Y., Swanson, J. A. y Cheng, H. (2011). State of research on giftedness and gifted education: A survey of empirical studies published during 1998-2010. Gifted Child Quarterly, 55(2), 126-138. https://doi.org/10.1177/0016986210397831

Elices Simón, J. A., Palazuelo Martínez, M. y del Caño Sánchez, M. (2006). El profesor, identificador de necesidades educativas asociadas a alta capacidad intelectual. Faísca, 11(13), 23-47. https://sid.usal.es/idocs/F8/ART21122/elices simon.pdf 
Feldhusen, J. F. (1997). Educating teachers for work with talented youth. En N. Colangelo y G. A. Davis (Eds.), Handbook of Gifted Education (2. ${ }^{a}$ ed., pp. 229-237). Allyn \& Bacon.

Geake, J. G. y Gross, M. U. M. (2008). Teachers'negative affect toward academically gifted students: An evolutionary psychological study. Gifted Child Quarterly, 52(3), 217-231. https://doi. org/10.1177/0016986208319704

Ghassib, H. B. (2010). Where does creativity fit into a productivist industrial model of knowledge production?. Gifted and Talented International, 25(1), 13-19. https://doi.org/10.1080/1533 $\underline{2276.2010 .11673540}$

Guerra Castillo, M., Alcalá Ruiz, B. M del S. y Ortiz González, G. (2014). Iniciativa con proyecto de decreto que reforma diversos artículos de la Ley General de Educación, en materia de educación especial para alumnos con sobrecapacidad intelectual. Senado de la República, México.

Kanevsky, L. (2011). Differential differentiation: What types of differentiation do students want? Gifted Child Quarterly, 55(4), 279-299. https://doi.org/10.1177/0016986211422098

Lewis, E. y Milton, M. (2005). Attitudes of teachers before and after professional development. Australasian Journal of Gifted Education, 14(1), 5-14.

Mandelman, S. D., Tan, M., Aljughaiman, A. M. y Grigorenko, E. L. (2010). Intellectual giftedness: Economic, political, cultural, and psychological considerations. Learning and Individual Differences, 20(4), 287-297. https://doi.org/10.1016/j.lindif.2010.04.014

Mettrau, M. B. (2010). Programas académicos para la formación de profesores de alumnos con superdotación y talento. Revista Electrónica Interuniversitaria de Formación del Profesorado, 13(1), 159-167. https://dialnet.unirioja.es/servlet/articulo?codigo=3163551

Mills, C. J. (2003). Characteristics of effective teachers of gifted students: Teacher background and personality styles of students. Gifted Child Quarterly, 47(4), 272-281. https://doi. org/10.1177/001698620304700404

Morales Silva, S. (2008). Programa de enriquecimiento en lectura para adolescentes talentosos. Revista de Psicología, 26(1), 93-122. https://doi.org/10.18800/psico.200801.005

National Association for Gifted Children. (2010). Redifining giftedness for a new century: Shifting the paradigm. http://www.nagc.org/sites/default/files/Position\%20Statement/ Redefining\%20Giftedness\%20for\%20a\%20New\%20Century.pdf

National Association for Gifted Children. (2013a). Advanced standards in gifted education teacher preparation. http://www.nagc.org.442elmp01.blackmesh.com/sites/default/files/ standards/Advanced\%20Standards\%20in\%20GT\%20(2013).pdf 
http://doi.org/10.15359/ree.25-3.15

http://www.una.ac.cr/educare

educare@una.ac.cr

National Association for Gifted Children. (2013b). Teacher preparation standards in gifted and talented education. http://www.nagc.org/sites/default/files/standards/NAGC-\%20 CEC\%20CAEP\%20standards\%20\%282013\%20final\%29.pdf

Organización para la Cooperación y el Desarrollo Económicos. (2010). Improving schools: Strategies for action in Mexico. Autor. https://doi.org/10.1787/9789264087040-en

Plunkett, M. y Kronborg, L. (2011). Learning to be a teacher of the gifted: The importance of examining opinions and challenging misconceptions. Gifted and Talented International, 26(1-2), 31-46. https://doi.org/10.1080/15332276.2011.11673587

Robinson N. M. (2008) The social world of gifted children and youth. En S. I.Pfeiffer (Ed.), Handbook of giftedness in children. Psychoeducational theory, research, and best practices (pp. 3351). Springer. https://doi.org/10.1007/978-0-387-74401-8_3

Tischler, K. y Vialle, W. J. (2009). Gifted students' perceptions of the characteristics of effective professors. En D. Wood (Eds.), The gifted challenge: Challenging the gifted (pp. 115-124). NSWAGTC.

Vialle, W. y Quigley, S. (2002). Does the teacher of the gifted need to be gifted? Gifted and Talented International, 17(2), 85-90. https://doi.org/10.1080/15332276.2002.11672992

Vialle, W. y Quigley, S. (2003). The teachers we want: Exploring the views of gifted students. En F. J. Mönks y H. Wagner (Eds.), Development of human potential: Investment into our future (pp. 125-128). Bock.

Vrignaud, P. (2006). La scolarisation des enfants intellectuellement précoces en France: Présentation des différentes mesures et de résultats de recherches. Bulletin de Psychologie, 5(485), 439-449. https://doi.org/10.3917/bupsy.485.0439

Whitlock,M.S.yDuCette,J.P.(1989).Outstanding andaverageteachers of thegifted:Acomparative study. Gifted Child Quarterly, 33(1), 15-21. https://doi.org/10.1177/001698628903300103

Whitton, D. (1997). Regular classroom practices with gifted students in grades 3 and 4 in New South Wales, Australia. Gifted Education International, 12(1), 34-38. https://doi. org/10.1177/026142949701200107

World Health Organization (2009). More than words. Conceptual framework for the international classification for patient safety. Version 1.1 (Final Technical Report). Autor. https://www.who. int/patientsafety/taxonomy/icps full report.pdf 